Thr ee di mensi onal anal ysi s of a cyl inder-type fl ux concent rat i on appar at us

\begin{tabular}{|l|l|}
\hline 著者 & Yoshi not o T. , Yamada Sot oshi , Bessho Kazuo \\
\hline $\begin{array}{l}\text { j our nal or } \\
\text { publ i cat i on ti t l e }\end{array}$ & I EEE Transact i ons on Magget i cs \\
\hline vol une & 24 \\
\hline number & 6 \\
\hline page r ange & $3150-3152$ \\
\hline year & $1988-11-01$ \\
\hline URL & ht t p: //hdl . handl e. net /2297/48317 \\
\hline
\end{tabular}




\title{
THREE DIMENSIONAL ANALYSIS OF A CYLINDER-TYPE FLUX CONCENTRATION APPARATUS
}

\author{
T. YOSHIMOTO, S.YAMADA AND K.BESSHO
}

\section{ABSTRACI}

This paper deals with three dimensional $A-\phi$ analysis of a newly developed cylinder-type flux concentration apparatus. We have already examined and analyzed several cypes of such apparatus, which utflize the flux concentration effect of eddy currents. [i][2][3] The new model treated here, attains compactness in size and high efficiency in flux concentration by assembling a conducting plate just iriside one or several excitation windings.

A new four-component direct finite element calculation method is applied to the present analysis, and three dimensional distributions of the flux density; the eddy current and the scalar potential are obtairled successfully. The divided direct calculation method is discussed in comparison with our former iterative. method.

\section{FLUX CONCENTRATION APPARATUS}

An ac flux concentration apparatus, which has been investigated at Kanazawa University since 1981 [4], has changed 1ts style three thes. The first fundamental model is the one with two conducting plates placed in parallel between a pair of ac excited colls. The second model is made by placing two more plates fust onto the plates of the first one in an orthogonal direction. The present third model is a circular one with some ac colls positloned around one conducting plate. This new type attaing compactness in size, ss well as high efflciency in flux concentration. Newly-built rims of the conductor for excitation windIngs enhance the flux concentration effect. In each model, flux concentration appears in the central air part due to the lnduced eddy currents in the conductor.

\section{DIVIDED DIRECT CALCULATION METHOD}

In order to analyze the phenomenon caused by eddy currents clrculating in a finfte conductor, three dimensional finte element $A-\phi$ method is indiapensable. However, the computer memory necessary for the analysis becomes huge because 4 components are required per node, In addition to the 3-D structure.

In addition, the induced scalar potential makes the system matrix not only huge, but also presents a problem concerning the interpretation of its nature. The role of the scalar potential has been discussed for a long time $[5][6][7]$ and many analysts have considered it to be caused from stored electrical charges as in the electric fleld. They might have cosidered it to be so because the dimension is the ame as che electrical potential and because it has never been calculated for the eddy current diffusion ptoblem due to huge size of the system matrix.

In 1986, we solved the four-component system matrx as it is by using an iterative method. [1] In 1987, we clariffed the role of the scalar potential by discussing its distribution.[2][3] Th1s time we develop a divided direct calculation method for two reasons. One is that the iterative method is too

Manuscr1pt recelved July 15, 1988

Takesh1 Yosh1moto is with the Department of Electrical Engineering. Ishikawa College of Technology. Tsubata, Ishikawa, 929-03 JAPAN. Sotosh1 Yamada and Kazuo Bessho are with the Electrical Energy Conversion Laboratory, Kanazawa Universicy, Kodatsuno, Kanazawa, 920 JAPAN. t1me-consuming and the other is that much experience is needed for selecting the convergent coeffictent.

Moreover, it may be added that the general time sharing system of the large-sized computer is enlarging the memory assigned for an Individual user, with the development of the computer technology in capacity and speed.

The divided direct calculation method uses the concept of the Gauss elimination method and two divided calculations for each variable of $A_{x} \cdot A_{y}, A_{z}$, whth one time calculation for $\phi$. The diviston of variables makes the more memory-saving possible. The selection of the number of the division should be considered in connection with the allowed computer memory.

\section{FIELD ANALYSIS EOUATIONS}

For a three dimensional, quasi-statfonary, eddy current diffusion problem, Maxwell's electromagnetic fleld equations together with the definition of the magnetic vector potential are stated as follows:

$$
\begin{aligned}
& \operatorname{rot} \overline{\mathrm{H}}=\overline{\mathrm{J}}_{s}+\bar{J}_{e} \\
& \operatorname{rot} \overline{\mathrm{E}}_{\mathrm{e}}=-\mathrm{j} \omega \overline{\mathrm{B}} \\
& \operatorname{div} \bar{B}=0 \\
& \operatorname{div} \bar{D}=\rho \\
& \vec{B}=\mu \bar{H} \\
& \bar{J}_{e}=o \overline{\mathrm{E}}_{e} \\
& \bar{B}=\operatorname{rot} \bar{A}
\end{aligned}
$$

where, $\vec{J}_{s}$ and $\vec{J}_{e}$ are the 1mpressed current and the induced eddy current densities respectively. Combining these equations shown above, we obtain two fundamental equations' for field analysis, under the assumption of constant $\mu$ in the $x, y$, and $z$ directions.

$$
\begin{aligned}
& \frac{1}{\mu} \nabla(\nabla \bar{A})-\frac{1}{\nu} \nabla^{2} \bar{A}-\sigma(j \omega \bar{A}+\nabla \phi)+\bar{J}_{g} \\
& \operatorname{div}\{\sigma(j \omega \bar{A}+g \operatorname{rad} \phi)\}=0
\end{aligned}
$$

From(9), the vector potential $\bar{A}$ is replaced as

$$
\nabla \bar{A}=-\nabla^{2} \phi / j \omega
$$

Ușing (10), (8) can be decomposed into three axiscomponent equations as shown in (11)-(13). Adding (9), the following four equations should be solved simultaneously.

$$
\begin{aligned}
& -\frac{1}{\mu} \nabla^{2} A_{x}+j \omega \sigma A_{x}=J_{s x}-(\sigma \nabla \phi)_{x}+\frac{1}{j \omega \mu} \frac{\partial}{\partial x}\left(\nabla^{2} \phi\right) \\
& -\frac{1}{\psi} \nabla^{2} A_{y}+j \omega \sigma A_{y}=J_{s y}-(\sigma \nabla \phi)_{y}+\frac{1}{j \omega \mu} \frac{\partial}{\partial y}\left(\nabla^{2} \phi\right) \\
& -\frac{1}{\mu} \nabla^{2} A_{z}+j \omega \sigma A_{z}=J_{s z}-\left(\sigma \nabla_{\phi}\right)_{z}+\frac{1}{j \omega \nu} \frac{\partial}{\partial z}\left(\nabla^{2} \phi\right) \\
& \nabla \cdot\left(\sigma j \omega\left(A_{x} \bar{u}_{x}+A_{y} \bar{u}_{y}+A_{z} \bar{u}_{z}\right)+\sigma \nabla \phi\right)=0
\end{aligned}
$$

The finite element formulation used here, is based on a triangular prism element and the Galerkin method. The structure of the combined global system matrix is shown in Fig.1. It seems not only huge due to the four components, but also sparse due to the Introduction of 
the scalar potential. The bandw1dth is $3 N+M$, if the total number of nodes and the bandwidth are assumed to be $N$ and $M$, respectively, per each component of $\bar{A}$.

Our divided direct method avoids zero submatrices unnecessary for the calculation, using the concept of the Gauss ellmination method. Both of the forward elimination process and the backward substitution process, are divided into four stages. In the forward elimination process, at the f1rst stage submatrices of $A A, S A$ and $P P$ are created in the main processor, at the second, submatrices of $B B, S B$ and $P P$ are treated, at the third, submatrices of $C C, S C$ and $P P$ are treated in the main processor and so on. As to the backward substitution process, at the first stage only PP, at the second, $S C$ and $C C$, at the third, $S B$ and $B B$, at the last stage, $S A$ and $A A$ are mantpulated in the main processor.

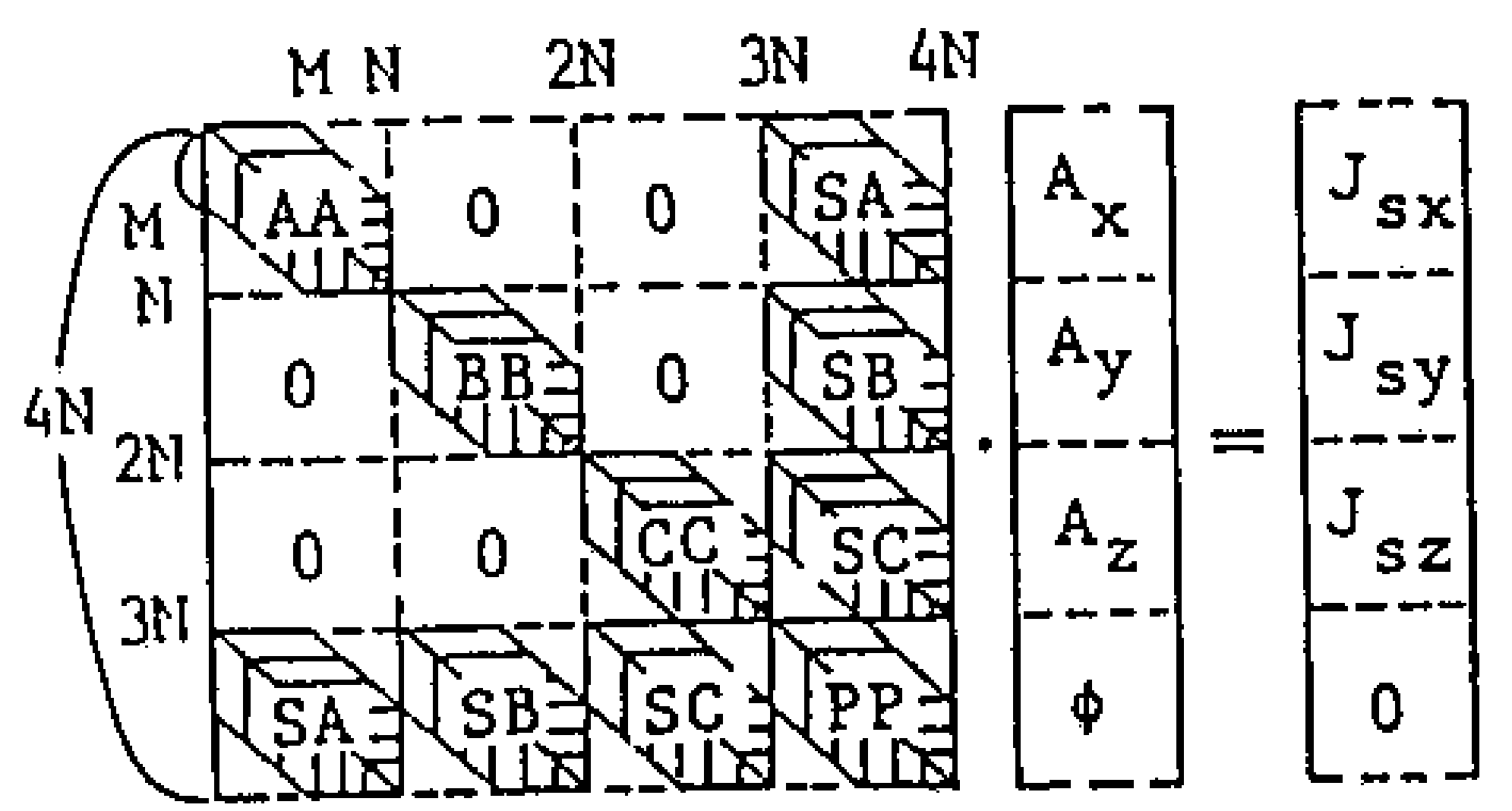

F1g.l Structure of Global Sysf̧em Matrix AIR-SLIT
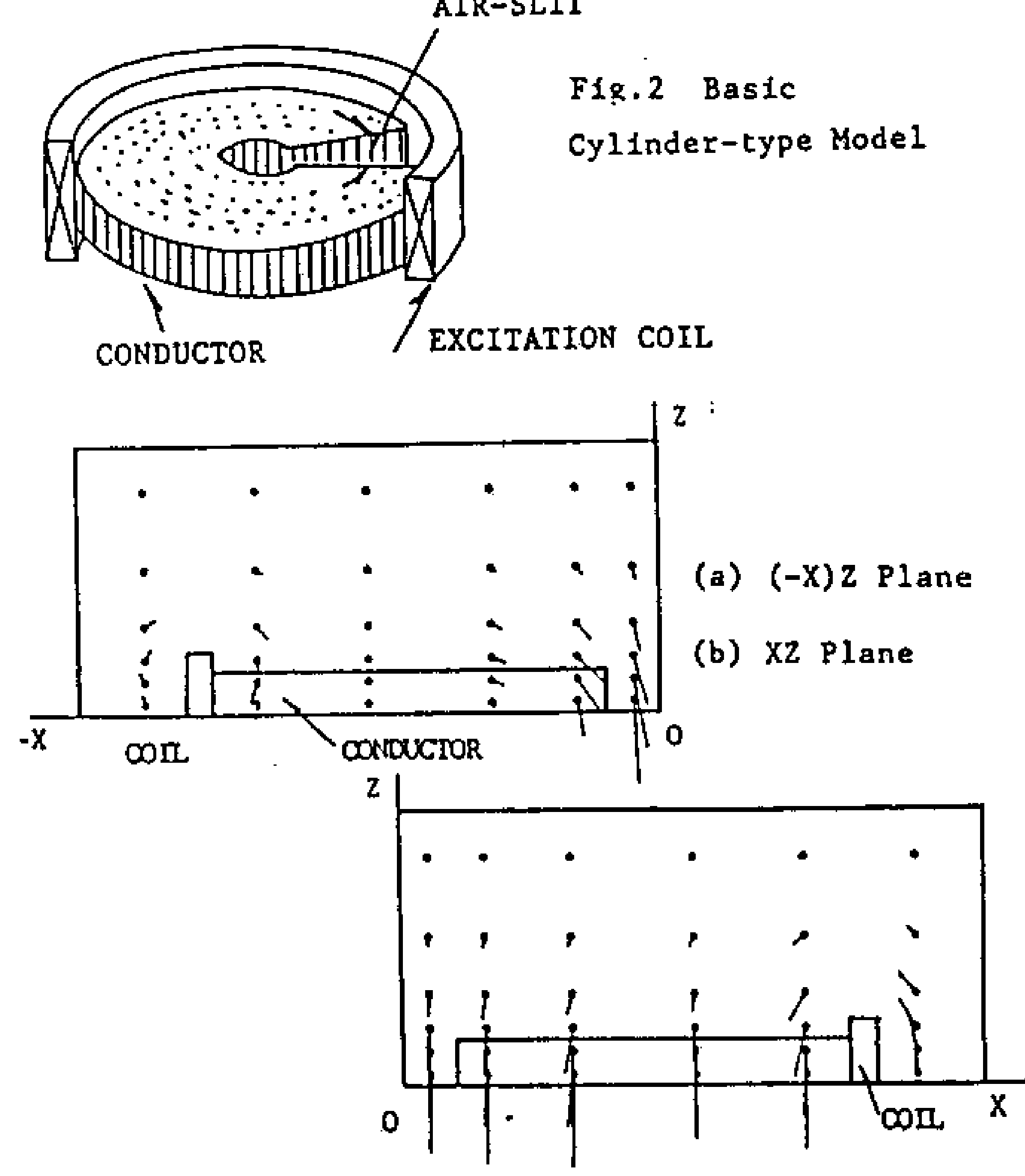

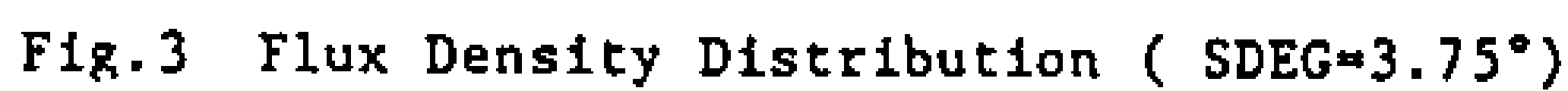

\section{BASIC CYLINDER-TYPE MODEL}

Fig.2 shows a basic model of the cylinder-type flux concentration apparatus. It consists of one or several excitation colls and one conductor with a tiny hole and one alr-slit in the radial direction.

Ac excitation induces eddy currents in the conductor, which results in concentrating the flux in the central hole. The model attains higher efficlency and makes the structure more compact, compared with the former ones. The reason is because existence of excitation windings fust around the conducting plate makes the flux linkage between a conductor and colls denser.

Fig.3-5 shows the results for the model of slit ange NE=858 $\quad 3$ shows distributions of the flux density $N E=858$. Fig. 3 shows dis for XZ plane. It is observed (a) the flux is reflected over the conductor and that the flux lo $X Y$ plane along the air-slit. Fig.4 is the distribution of the eddy current density in the is the distre. Fig. 5 shows the circumferential districentral plane. Fis.s potential. It serves the eddy currents to circulate in the conductor.
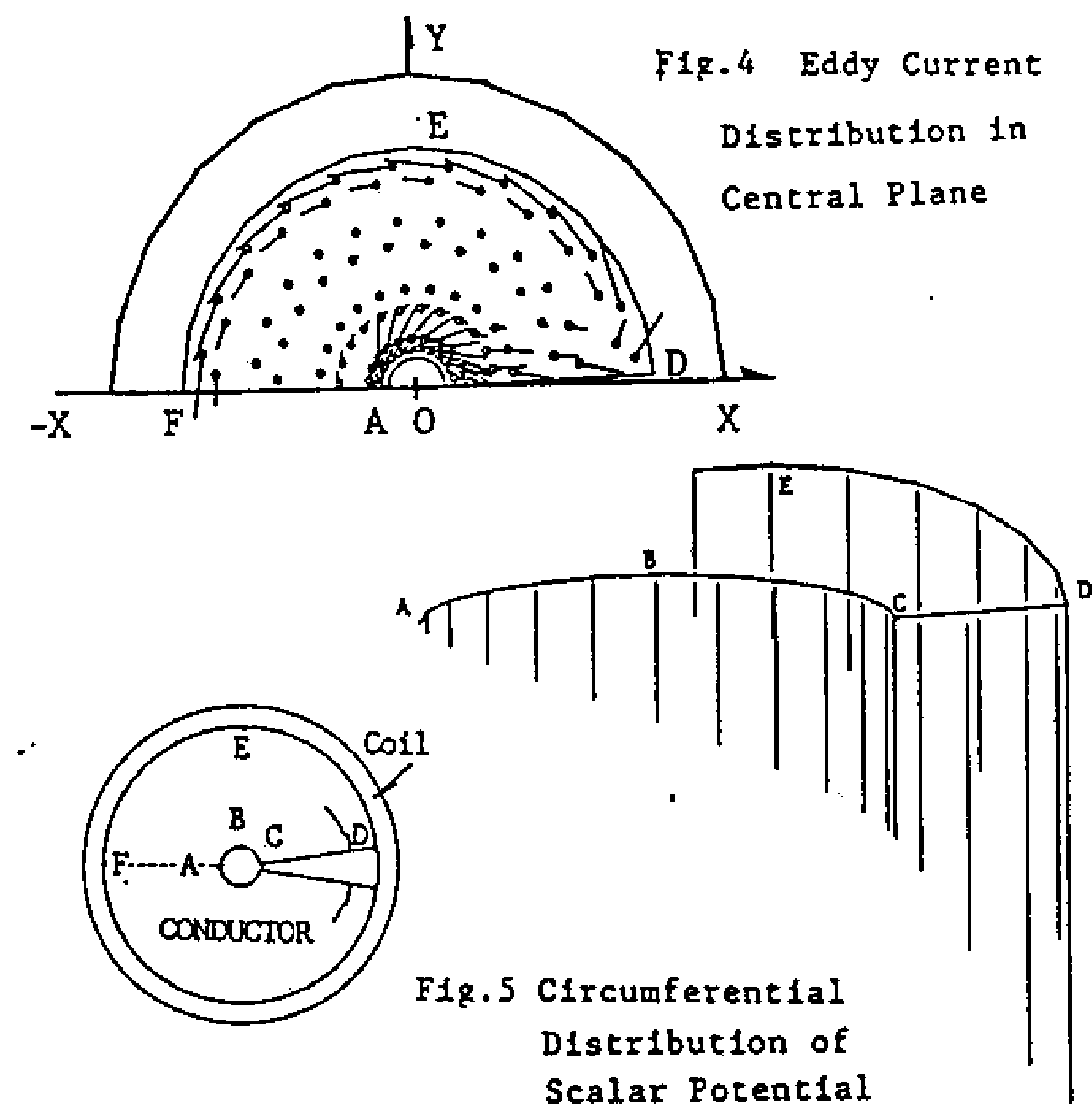

MODEL' NITH MULTI-RIMS

In order to 1mprove the degree of flux concentration, several rims and multt-windings are found effective. Fig.6 shows the relation between the number of windings and the factor of flux concentration (or flux density). FEM calculation is made on the model with three rims and two windings as shown in Fig.7. One fourth of the model of total nodes NP=1098 and total elements $N E=1625$ is analyzed. The Dirichlet boundary condition is tmposed on the outer boundary surface with zero vector potential along z-axis.

As for the boundary condition of the scalar potential, zero values are set on the $(-x) Z$ plane. Frequency of the impressed current is $60 \mathrm{~Hz}$, the conductivity of the copper plate $8.62 \times 10^{7} \mathrm{~s} / \mathrm{m}$, the width of the slit 3.0 $\mathrm{mm}$, and the ampere turn of the coll $2.0 \times 10^{-12} \mathrm{AT} / \mathrm{mm}^{2}$.

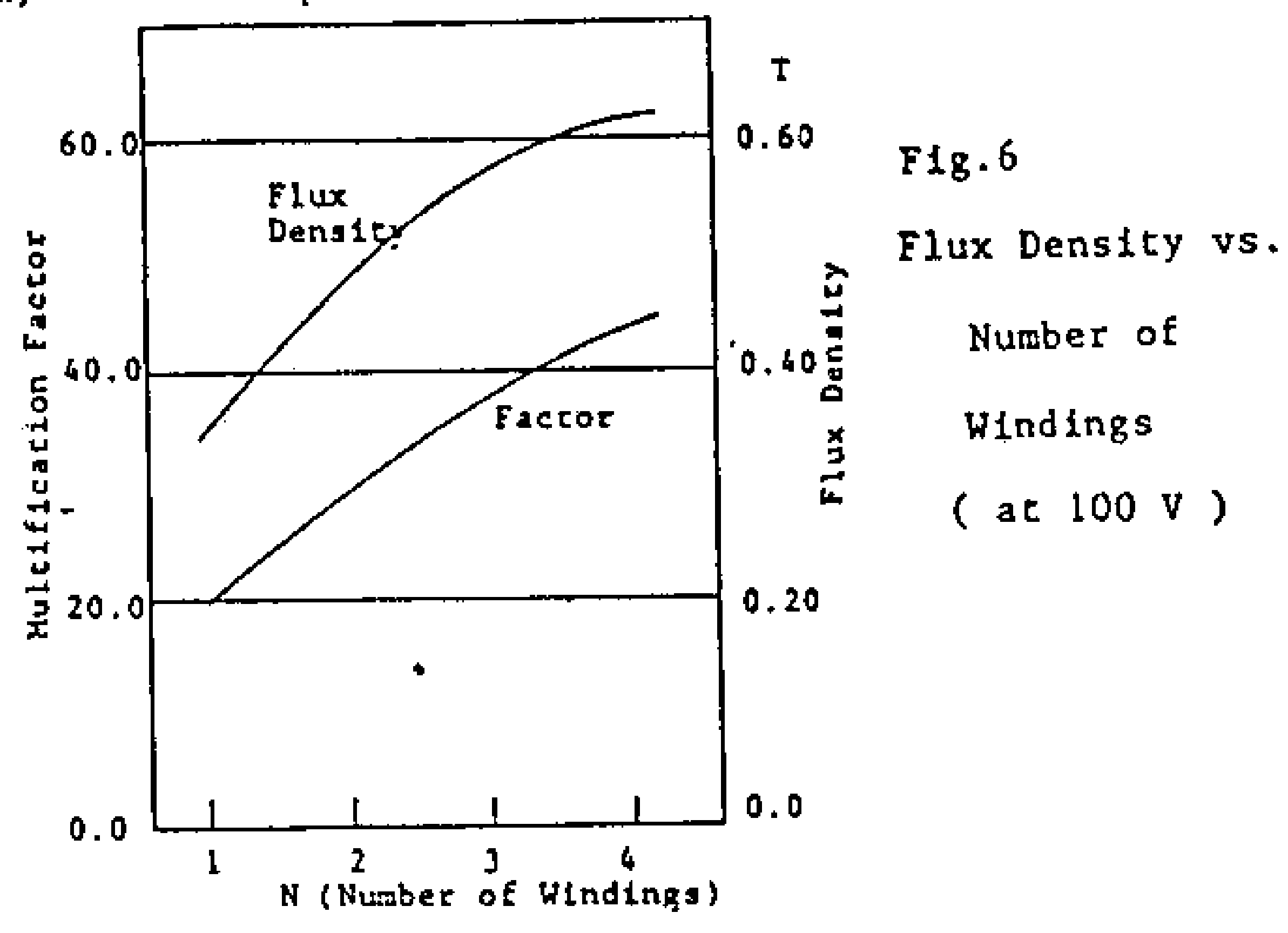




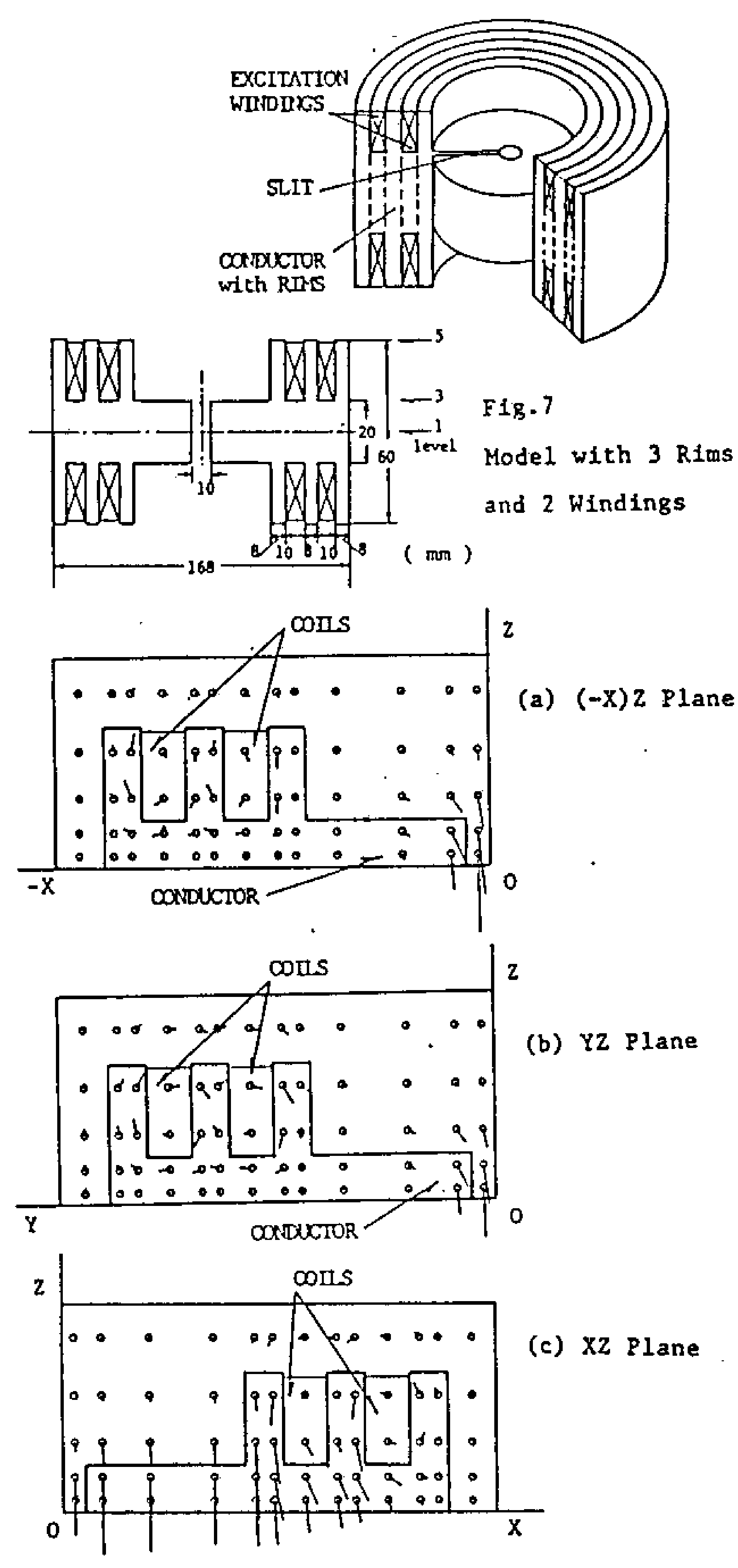

Fig.8 Flux Density Distribution
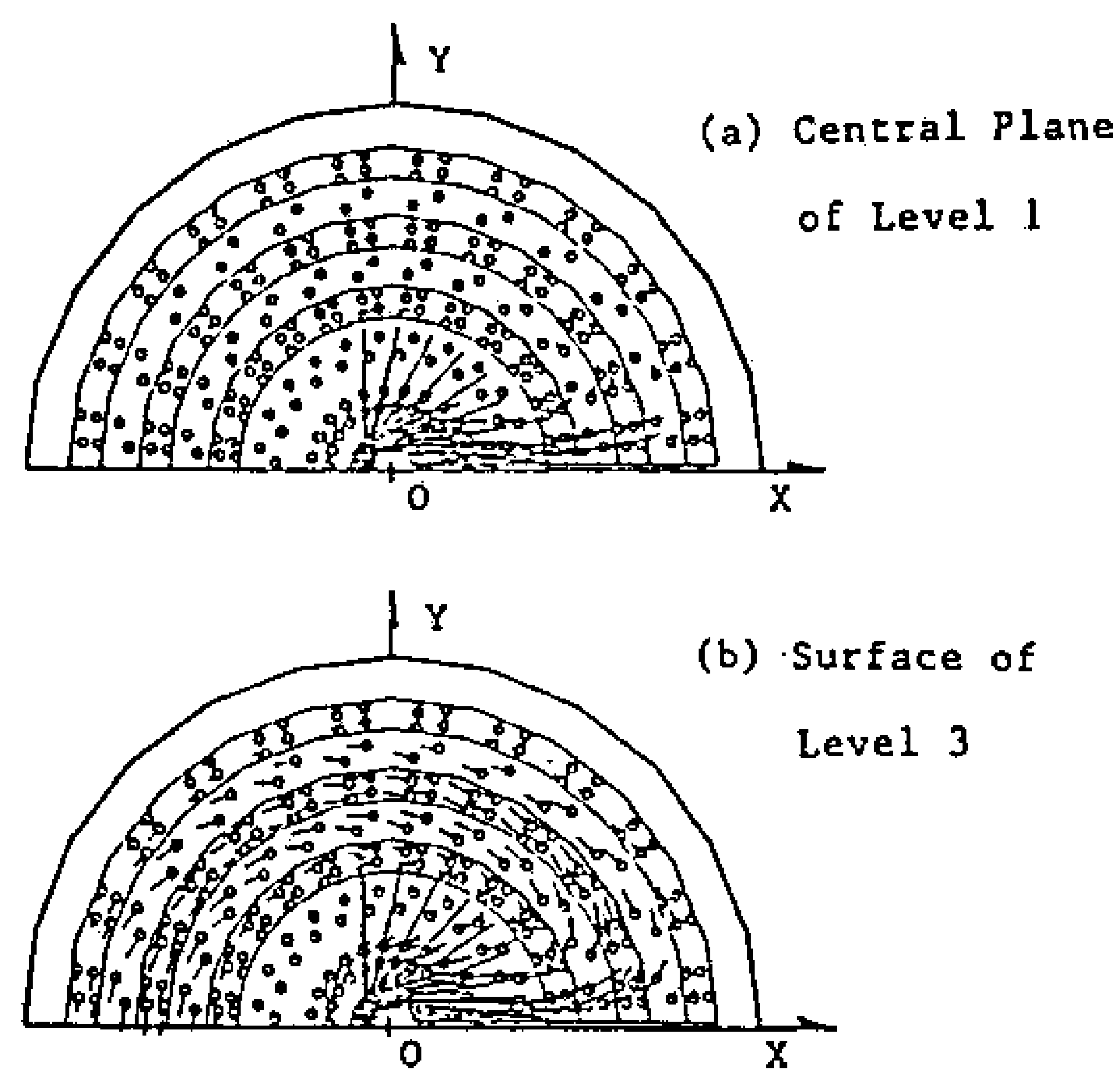

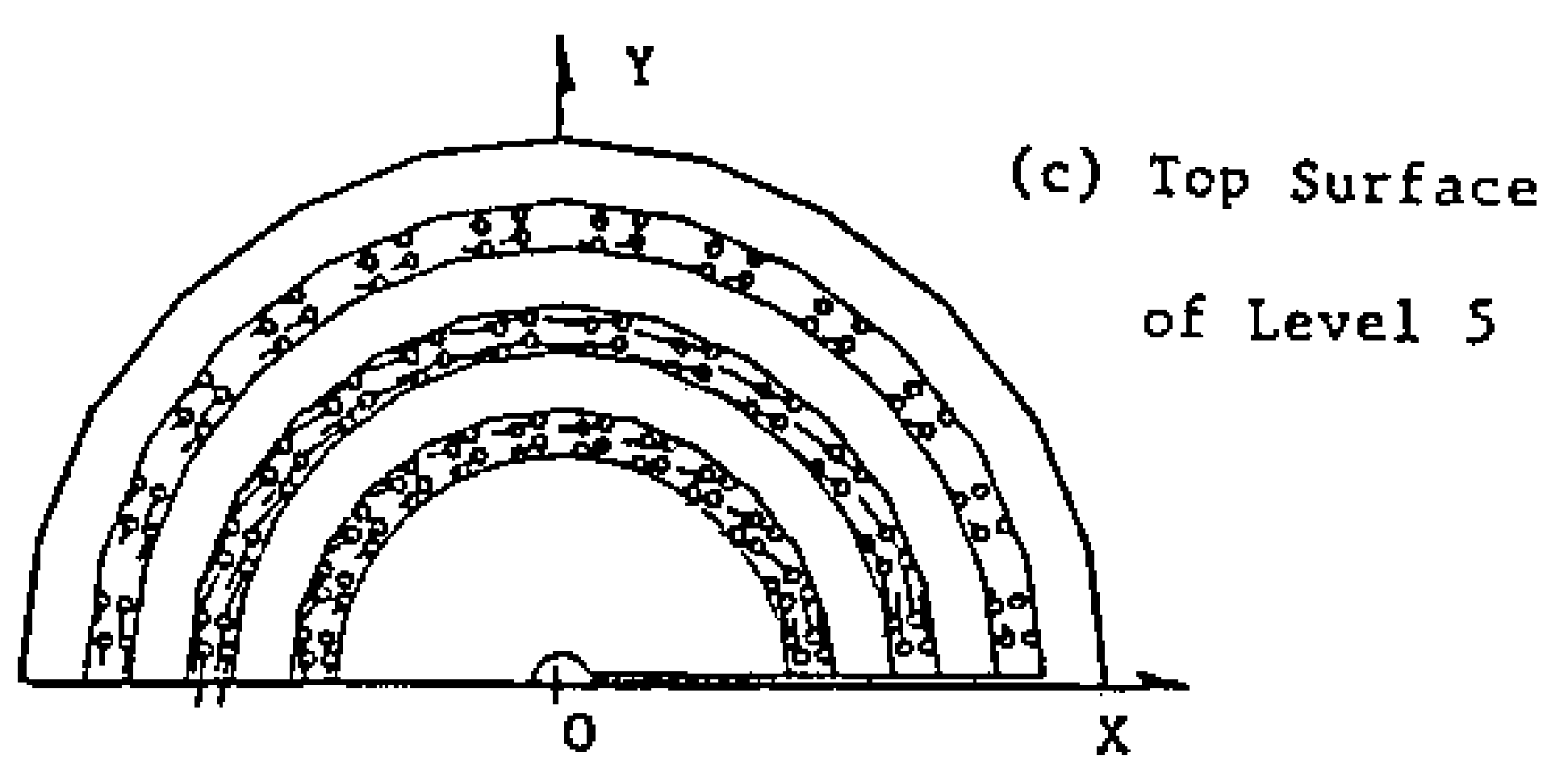

Fig.9 Eddy Current Distribution

Fig. 8 shows the distributions of the flux density, (a) for ( $X) Z$ plane, (b) for $Y Z$ plane, (c) for $X Z$ plane. It is also observed that the flux density is higher in the left stde in the hole. Fig. 9 shows the distributions of the eddy current density, (a) for the central plane of level 1 , (b) for the surface of level 3. (c) for the top surface of level 5 .

In this analysis, one fourth of the model should be treated, because of the existence of two symmetric nlanes. Every submatrix in this analysis comes to have the bandwidth of about two times as that in the case that one eighth region is treated. As a whole, this model is memory-consuming.

\section{CONCI.USION}

Two models of the cylinder-type flux concentration apparatus are analyzed by applying a newly developed 3-D divided direct method of calculation. In spite of the memory consuming model, we obtained the distributions of the flux density and the current density, as well as the scalar potential. It is observed that eddy currents concentrase in the Inner circumference of the conductor and contribute to strengthen the flux density in the hole, espectally in the left side. The distribution of the scalar potential is confirmed to correct the flow of the eddy current not so as to flow out of the conductor. It is also confirmed that multi-rins are usefull to intensify the flux concentration effect by Increasing the linkage of the magnetic field.

\section{REFERENCES}

[1] T.Yoshimoto et a1.,"3-D Finfte Element Analysis of Flux Reflection \& Flux Concentration Effects of Eddy Currents," IEEE Irans, on Magnetics, Vol.MAG22, No5, 834 (1986)

[2] T.Yoshimoto et al.,"3-D Distribution of Flux Density \& an Inference about Scalar Potential in a Flux Concentration Model," IEEE Trans.on Magnetics, Vol. MAG-23, No5, 3041 (1987)

[3] T.Yoshimoto et al. "Four Component 3-0 FEM Analysis of Flux Concentration Apparatus with Four Plates," IEEE Trans, on Magnetics, Vol.MAG-24,Nol,126 (1988)

[4] K.Bessho et al., "Assymmetrical Eddy Currents and Concentration Effect of Magnetic Flux in a High Speed Rotating Disc," IEEE Trans.on Magnetics, Vol. MAG-21, NoS (1985)

(5) C.S.Biddlecombe et al., "Methods for Eddy Current Computation in Three Dimensions," IEEE Trans.on Magnetics, Vol.MAG-18,No2 (1982)

[6] M.V.K.Char1 et al. "Three Component Two Dimensional Analysis of the Eddy Current Diffusion Problem," IEEE Trans.on Magnetics, Vol.MAG-20 , NoS (1984)

(7) W.Muller et al. "A Method for Numertcal Calculation of 3-D Eddy Currents," IEEE Trans.on Magnetics, Vol.MAG-21, No6 (1985) 